\title{
Backward-Auto-Regressive Model Based Rotor-bearing System Stability Prediction
}

\author{
Jiali Yang ${ }^{1 . a}$, Weimin Wang ${ }^{2 . b}$, Yongjiang Zhu ${ }^{3 . c}$, Ya Zhang ${ }^{4 . d}$ \\ Diagnosis and Self-recovery Engineering Research Center, Beijing University of Chemical \\ Technology, Beijing 100029, China \\ ayangjiali181989@126.com, bwwmbuct@163.com, 'zyj319205@126.com, \\ 'zhangya@mail.buct.edu.cn
}

\begin{abstract}
Keywords: damping ratio estimation, BAR model, singular value decomposition, rotor-bearing system
\end{abstract}

\begin{abstract}
This paper focus on rotor-bearing system parameter identification with impulse excitation in horizon and vertical which is based on Backward -auto-regressive model. Singular value decomposition is applied to reduce the noise and the proper AR model order and de-noising threshold are selected. In this paper, the damping ratio is identified within the different rotating speed and different impulse excitation, and the error is calculated within the different noise level and different AR model order when compared with the ideal model. Though the theoretical analysis, simulation analysis and experimental research, We can indicate that the BAR model has a good performance in system identification and elimination of false modal.
\end{abstract}

\section{Introduction}

In recent years, with the development of high parameter turbo-machinery, the instability failure caused by non-conservative tangential force which is excited by air flow becomes very prominent. Therefore, determining and forecasting the rotor instability which is based on the damping ratio changes of rotor-bearing system in turbo-machinery design and fault diagnosis has an important signification.

There are some system identification methods which are commonly used in time domain and frequency domain: least squares, random reduction, wavelet transform, EMA, and ARMA methods. For single-input single-output or multiple-input multiple-output rotor-bearing system, literature [1] introduced the origin theoretical basis, and the application of forward and backward ARMA method, then compared the advantages and disadvantages of these methods theoretically but did not have test improvements. Literature [2-8] described the methods for estimating the ARMA parameters, such as power spectrum, the residual estimates, EMA law. According to Akaike's Hongzhi AIC and BIC criteria [7-12], the applicability of the model is tested. However the accuracy of the results is decreased because of the noise interference, in recent years, the QR decomposition [9] and singular value decomposition [10-11] has proven to be an effective tool for minimizing the impact of noise.

This paper introduces the application of BAR (p) model in parameter identification on the real rotor - bearing system, using singular value decomposition method to reduce false-modal interference, through numerical simulation and experimental validation, the BAR model, in the process of damping ratio estimation, can effectively eliminate the false mode with high accuracy.

\section{BAR model and parameter identification}

Single output BAR model. Horizontal or vertical impulses were applied to the rigid rotor system for damping estimation using a power hammer; the output signal is a continuous free decaying time sequence from an eddy current transducer. The sampling frequency is $1024 \mathrm{~Hz}$, and then we can achieve a discrete time sequence $\left\{\mathrm{y}_{t}\right\}$ for BAR modeling which is required to be a smooth normal zero mean time sequence. Extract trend term and zero-treatment are applied [12]. 
For single-input single-output rotor system, it's BAR (p) model is expressed in reversed time direction, the captured response signal $y_{n}$, with $\mathrm{N}$ samples takes the form [1]:

$$
y_{n}=-b_{1} y_{(n+1)}-b_{2} y_{(n+2)}-\cdots-b_{p} y_{(n+p)}+\omega_{n}=-\sum_{i=1}^{p} b_{i} y_{(n+i)}+\omega_{n}
$$

where the sample interval is $\Delta \mathrm{t}$ and $\omega_{n}$ is a zero mean Gaussian white noise sequence, and $b_{i}$ is BAR model parameter, $\mathrm{p}$ is the BAR model order.

Bring in z-domain polynomial:

$B_{(z)}=1+b_{1} z^{1}+b_{2} z^{2}+\cdots+b_{n} z^{n}$

Backward prediction may appear counterintuitive, however it should be remembered that the block of $\mathrm{N}$ date samples has already been obtained, the following set of linear prediction equation using the entire sequence takes the form [10]:

$$
\left(\begin{array}{cccc}
y_{(1)} & y_{(2)} & \cdots & y_{(p)} \\
y_{(2)} & y_{(3)} & \cdots & y_{(p+1)} \\
\vdots & \vdots & \ddots & \vdots \\
y_{(N-p)} & y_{(N-p+1)} & \cdots & y_{N-1}
\end{array}\right)\left(\begin{array}{c}
b_{1} \\
b_{2} \\
\vdots \\
b_{p}
\end{array}\right)=-\left(\begin{array}{c}
y_{(0)} \\
y_{(1)} \\
\vdots \\
y_{(N-p-1)}
\end{array}\right)
$$

After solving for these discrete poles $\mathrm{z}_{\mathrm{i}}=\mathrm{e}^{\lambda_{\mathrm{i}}{ }^{*} \Delta \mathrm{t}}$. the parameter estimation is completed and the damping ratio is achieved using $\xi_{i}=-\operatorname{re} a l\left(\lambda_{i}\right) /\left|\lambda_{i}\right|$.

SVD and choice of de-noising threshold. In the z-domain, the equation (1) becomes:

$$
Y(z)=1 /\left(1+\sum_{i=1}^{p} b_{i} z^{i}\right) * W(z)=1 / B(z) * W(z)
$$

However, for the real rotor-bearing system, the input signal is hardly to be a pure zero mean Gaussian white noise, so there is a conversion between the impulse input and the white noise signal:

$$
X(z) \rightarrow \frac{A(z)}{B^{\prime}(z)} \rightarrow W(z) \rightarrow \frac{1}{B(z)} \rightarrow Y(z)
$$

Therefore, the actual value of zero poles obtained consists of three aspects, one is noise modal caused by noise, another one is spurious modes due to the calculation, and the other is the system true modal. The first two will reduce the accuracy and should be eliminated using SVD [10]:

$$
Y=U \Sigma V^{T}
$$

where $\mathrm{U}$ and $\mathrm{V}$ are real unitary matrices whose columns are designated left and right singular vectors, and $\Sigma$ takes the form:

$$
\Sigma=\left[\begin{array}{cccc}
\sigma_{1} & 0 & \cdots & 0 \\
0 & \sigma_{2} & \cdots & 0 \\
\vdots & \vdots & \ddots & \vdots \\
0 & 0 & \cdots & \sigma_{\mathrm{n}}
\end{array}\right], \sigma_{1} \geq \sigma_{2} \geq \cdots \geq \sigma_{\mathrm{n}}
$$

If the matrix $Y$ is noiseless, the rank of $\Sigma$ is less than $n$, with noise added, $Y$ achieves full rank. Therefore, matrix $\mathrm{Y}$ is consisted of two sub-matrix, one stands for signal message, and another one stands for noise [11].

$$
Y=U \Sigma V^{T}=U\left[\begin{array}{cc}
\Sigma \sigma_{r} & 0 \\
0 & \Sigma \sigma_{r+}
\end{array}\right] V^{T}=\left[\begin{array}{cc}
U \Sigma \sigma_{r} V^{T} & 0 \\
0 & U \Sigma \sigma_{r+} V^{T}
\end{array}\right]=\left[\begin{array}{cc}
Y_{1} & 0 \\
0 & Y_{2}
\end{array}\right]
$$

As $Y_{1}<<Y_{2}, Y_{2}$ is abandoned as noise matrix by setting appropriate de-noising threshold $\mathrm{r}$, Good success has been obtained using the following ratio criterion [10]:

$$
\sqrt{\frac{\sigma_{1}^{2}+\sigma_{2}^{2}+\cdots \sigma_{r}^{2}}{\sigma_{1}^{2}+\sigma_{2}^{2}+\cdots \sigma_{n}^{2}}}>0.99
$$


Choice of BAR model order.Order overspecification, where $p$ is set to be greater than actual number, improves the identification accuracy of both frequency and time domain methods where measurement noise exists. However, it also creates a problem of how to distinguish the noise-related poles from the true physical or signal poles. There is Akaike's Hongzhi AIC and BIC criteria [7]:

$$
A I C=N \log \left(\sigma^{2}\right)+2 p
$$

In general, for the simple calculation, $p=N / 2$ [10].

\section{Simulation and experiment}

Simulation. A rotor-bearing system with a single disk supported by two sliding bearings can be simplified as a mass-spring-damper system. Assuming the damping ratio is 5\%, natural frequency is $300 \mathrm{~Hz}$, the free decaying response with $2 \%$ noise and 5\% noise is obtained in figure 3.1 .
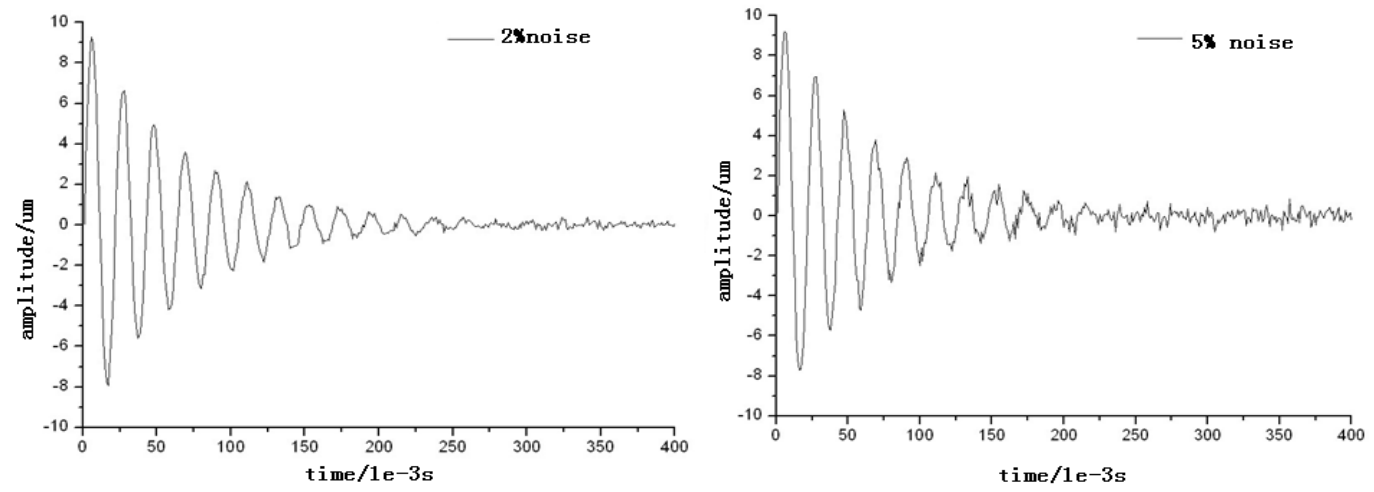

Fig. 1. Free decaying response with $2 \%$ noise and $5 \%$ noise

After SVD, all the poles associated with physical signals to be on or outside the z-domain unit circle, poles associated with noise are located inside the unit circle, figure 3.1 illustrates the noise pole filtering ability of BAR formulation.

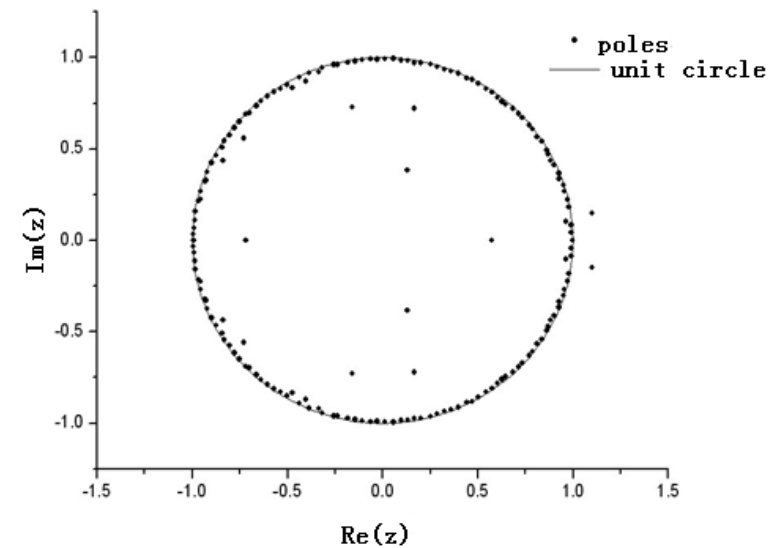

Fig. 2. All signal and noise poles in the z-plane

As is mentioned previously, AR model order and de-noising threshold has a great effect on accuracy. So in this paper, the influence of different order and de-noising threshold is discussed. 

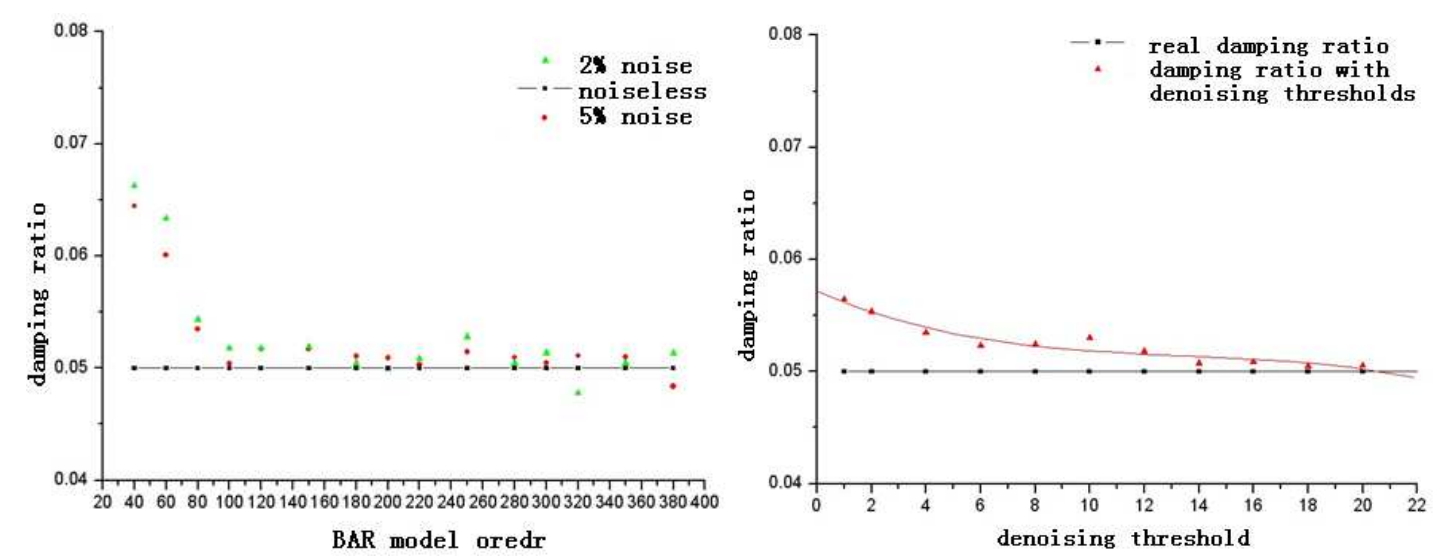

Fig. 3. The damping ratio under the different AR model order and de-noising threshold

According to figure 3.3 , when $\mathrm{p}<220$, with the increasing of AR model order, the damping ratio is closer to the ideal value. While $\mathrm{p}>220$, the larger the $\mathrm{p}$ is set, the larger the error is obtained. When $\mathrm{p}=200, \mathrm{r}<20$, with the increasing of de-noising threshold, the damping ratio is closer to the ideal value. The error is show as follows:

Table 1 The error under the different model order and de-noising threshold

\begin{tabular}{|c|c|c|c|c|c|}
\hline $\mathrm{f} \quad \mathrm{p}$ & 50 & 100 & 150 & 200 & 250 \\
\hline 1 & 0.1433 & 0.0536 & 0.0254 & 0.0108 & 0.0015 \\
\hline 5 & 0.2692 & 0.0428 & 0.0518 & 0.0290 & 0.0356 \\
\hline 10 & 0.0909 & 0.0748 & 0.0288 & 0.0522 & 0.03 \\
\hline 15 & 0.49336 & 0.2350 & 0.0966 & 0.0424 & 0.0307 \\
\hline
\end{tabular}

Experiment. The shaft with the length of $560 \mathrm{~mm}$ and diameter of $10 \mathrm{~mm}$, was supported by two sliding bearings, the disk is located in the middle of the shaft with the mass of $0.8 \mathrm{~kg}$. The impulse is applied to the rotor system when the shaft is on operating speed.
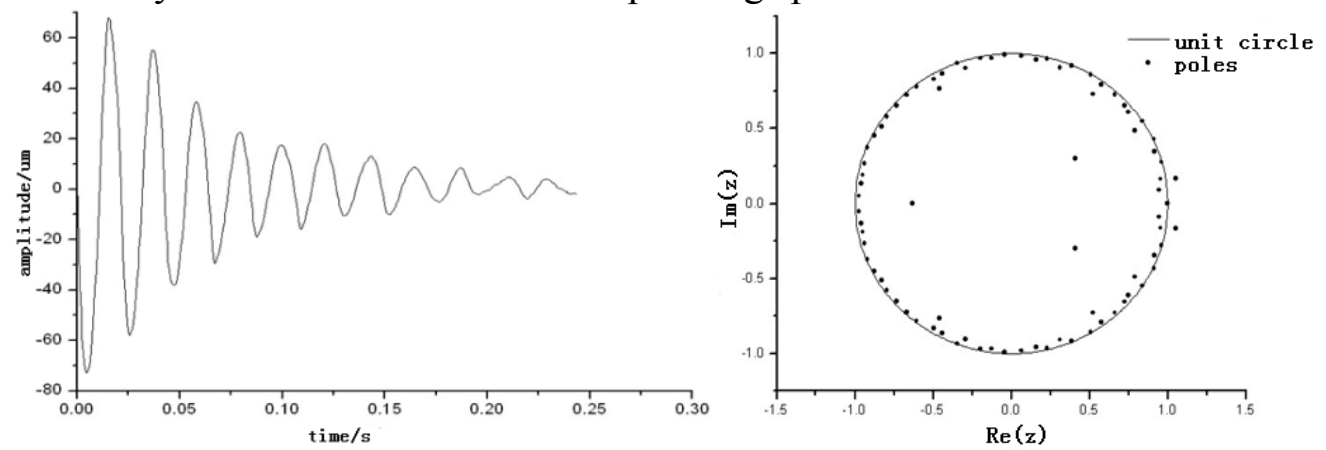

Fig. 4. Rotor probe waveform and poles in the z-plane due to horizontal impulse at $1500 \mathrm{rmp}$

With the increasing of the speed, the damping ratio is getting smaller, however, damping ratio increased nearby the resonance frequency where the vibration amplitude increased suddenly.

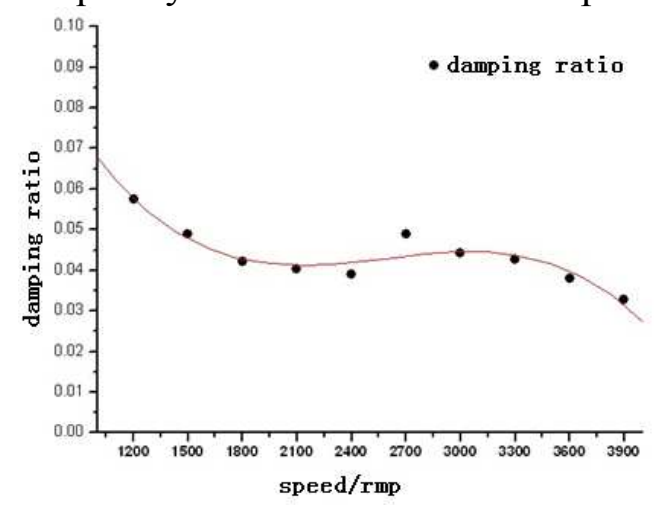

Fig. 5. The damping ratio under different speed 


\section{Summary}

For the BAR method, the input signal is not required, which may do a great advantage to some field project with unknown input signal. after SVD, setting an appropriate backward AR model order and de-noising threshold which have an great affect on parameter identification accuracy, the BAR model can distinguish the system poles and noise poles clearly in z-plane, combined with the fact that the AR model is accurate model, it can be concluded that the solutions, i.e. the system poles and eigenvectors, obtained by the backward AR model are more accurate. the result of the simulation and experiment indicates that the BAR model has a good performance in system identification and elimination of false modal.

\section{Acknowledgments}

This work was supported by the general Program of Natural Science Foundation of China (50975018).

\section{References}

[1] Ping Zhong, Rotor Bearing System Identification Using Time Domain Methods. PhD thesis, University of Virginia,1997.

[2] R.Douglas Martin, Victor J. Yohai. Robustness in Time Sequence and Estimating ARMA Models, University pf Washington, 1984

[3] Aydin Kizilkaya, Ahmet H.Kayran, ARMA model parameter estimation based on the equivalent MA approach ,J. Digital Signal Processing 16(2006) 670-681

[4] Palle Andersen. Identification of Civil Engineering Structures using Vector ARMA Models,J.Aalborg University,1997.

[5] Ralf Christ, Josef Steinebach. Estimating the Adjustment Coefficient in an ARMA(p,q) Risk Model, J. Mathematics and Economics 17(1995)149-161

[6] Qi-jiang Song, Han-Fu Chen.Identification of errors-in-variables systems with ARMA observation noises,J. System and Control Letters 57(2008) 420-424.

[7] Mark S. Voss, Xin Feng. ARMA Model Selection Using Particle Swarm Optimization and AIC Criteria,J. Marquette University, Milwaukee Wisconsin,2002

[8] Serena Ng, Pierre Perron. Unit Root Tests in ARMA Models with Data-Dependent Methods for the Selection of Truncation Lag,J. Journal of the American Statistical Association, Volume 90, Issue 429(1995),268-281.

[9] T.Patrik Nordberg, Ivar Gustafsson, Using QR factorization and SVD to solve input estimation problems in structural dynamics,J. Comput.Methods Appl.Mech.Engrg. 195(2006) 5891-5908

[10] Charles Hunter Cloud,Stability of Rotors Supported by Tilting Pad Journal Bearings, D. University of Virginia,2007.

[11] Tangbaoping, Jiangyonghua, Zhangxiangchun. The Method of Fault Feature Extracting Based on Singular Value Decomposition and Empirical Mode Decomposition Sliding Bearing, J. Chongqing University, China General Aviation Technology Institute, 2010

[12] Yangshuzi, Wuya, Xuanjianping. Time Sequence Analysis of the Engineering Applications ,M.Huazhong Technology University press, 2007. 